\title{
Matrix Metalloproteinase 9 for Evaluating Invasiveness and Recurrence in Pituitary Adenoma Patients: A Meta-analysis
}

\section{bowen sun}

Beijing Tongren Hospital https://orcid.org/0000-0002-0409-5559

\section{Congxin Dai}

Peking Union Medical College Hospital

Xiaohai Liu

Xuanwu Hospital

Renzhi Wang

Peking Union Medical College Hospital

Jun Kang ( $\nabla$ kangjuntr@163.com )

Research article

Keywords: MMP-9, Pituitary Adenoma, Invasion, Recurrence, Meta-analysis

Posted Date: February 19th, 2020

DOI: https://doi.org/10.21203/rs.2.24007/v1

License: (c) (1) This work is licensed under a Creative Commons Attribution 4.0 International License.

Read Full License 


\section{Abstract}

Background: In recent years, matrix metalloproteinase 9 (MMP-9) has been consideredto be an indicator of the aggressiveness and recurrence of pituitary adenomas, especially in China. However, some results have led to many conflicts. Therefore, it is necessary to perform a meta-analysis to obtain a clear correlation between MMP-9 and invasiveness and recurrence.

Methods: Eligible studies obtained through multiple searches were included, and the odds ratios (ORs) and hazard ratios (HRs) with 95\% confidence intervals (95\% Cls) were estimated. Funnel plots were used to evaluate publication bias. In the current meta-analysis, 23 articles with a total of 1,213 patients were included, including three studies conducted in Europe and China.

Results: High MMP-9 expression and invasiveness of pituitary adenomas $(\mathrm{n}=22, \mathrm{OR}=4.75,95 \% \mathrm{Cl}=$ 3.53-6.39, $p<0.05)$ and tumour recurrence $(n=3, O R=4.38,95 \% \mathrm{Cl}=1.76-10.94, p=0.002)$ were closely related. In addition, the expression of MMP-9 in patients with functional and non-functional pituitary adenomas was also significantly different $(n=3, O R=2.07,95 \% \mathrm{Cl}=1.03-4.16, p=0.041)$.

Conclusion: MMP-9 is closely related to the invasiveness, functional status and recurrence of pituitary adenoma and should be used as a routine diagnostic index for surgical treatment of pituitary adenoma.

\section{Background}

Pituitary adenomas account for approximately $15 \%$ of intracranial tumours and are listed as the second most common intracranial tumour ${ }^{[1,2]}$. Most pituitary adenomas are benign and do not exhibit swelling. However, approximately $30-40 \%$ of pituitary adenomas are highly invasive and can penetrate into surrounding tissues ${ }^{[3]}$. For many years, similar to the treatment of prolactinomas, surgical removal of pituitary adenomas has been the first choice for treatment. It is worth noting that complete removal of invasive pituitary adenomas is difficult due to the massive invasion of adjacent tissues such as the cavernous sinus and dura. Despite other clinical treatments, such as radiation therapy and alternative medical treatments, pituitary adenomas tend to relapse and are not sensitive to these therapies ${ }^{[4]}$. The current diagnosis of invasive adenoma is based on preoperative imaging, gross observation during surgery, and histopathology results after surgery. Surgeons still lack an accurate diagnostic biomarker to assess the aggressiveness or predict clinical outcomes of pituitary adenomas ${ }^{[5]}$. Although many studies have shown that pituitary adenomas are monoclonal in origin ${ }^{[6]}$, the pathogenesis and invasive progression of pituitary adenomas have not been clarified. Nevertheless, some biomarkers of pituitary adenoma invasiveness have been proposed ${ }^{[7,8]}$. However, these results have not been confirmed and are not generally accepted by all clinicians. We hope to predict the biological progress of tumours through molecular markers, which has potential application value for tumour diagnosis and targeted therapy. Therefore, it is necessary to identify a biomarker to manage aggressive pituitary adenomas and improve patient prognosis ${ }^{[9]}$. 


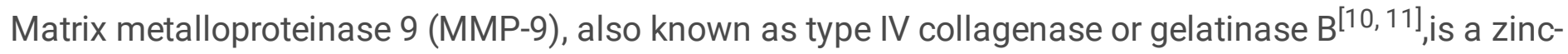
dependent protein. Due to its capacity for extracellular degradation, MMP-9 is thought to be involved in the aggressiveness of a variety of human malignancies such as breast ${ }^{[12]}$, prostate ${ }^{[13]}$, bladder ${ }^{[14]}$, and pancreatic cancer ${ }^{[15]}$. Previous studies have shown that the expression of MMP-9 in pituitary adenomas may contribute to the biological occurrence and development of pituitary adenomas, including the invasion, recurrence and spread of pituitary adenoma cells. Therefore, understanding the role of MMP-9 in the invasion and prognosis of pituitary adenomas provides a basis for developing effective clinical treatment strategies.

Many studies have suggested that MMP-9 expression may be involved in the occurrence and development of pituitary adenomas ${ }^{[16-18]}$. However, some reports have shown opposite results ${ }^{[19,20]}$. Based on these contradictions, we performed a meta-analysis to provide a more comprehensive and reliable conclusion regarding whether MMP-9 expression is correlated with human pituitary adenoma .

\section{Results}

\section{Search Results And Characteristics Of Studies}

The article retrieval strategy is shown in Fig. 1. Initially, 102 articles were generated through a structured literature search. After reviewing the title and abstract, 59 articles were excluded, of which 18 involved in vitro experiments, 35 were studies of other types of MMPs, and 6 were in vivo studies. Then, the remaining 43 articles were screened a second time, and 20 were excluded for the following reasons: 3 used continuous variables, 5 were retrospective, and 12 had insufficient data. However, only three studies were conducted in Europeans patients, and other studies were conducted in Chinese patients. Ultimately, 23 eligible articles were included.

Table 1 summarizes the general characteristics of the 23 studies. There were 1,213 patients with an average age of 42.2 years. Most studies were conducted in Chinese populations. The positive expression rate of MMP-9 was $49.0^{[19]}$ to $96.7 \%{ }^{[20]}$. Three studies provided only recurrence data ${ }^{[18,21,22]}$, and 23 studies provided invasion data. In addition, three articles provide functional status data ${ }^{[19,23,24]}$. IHC methods were used to detect MMP-9 protein in pituitary adenoma tissue. If the nucleus or cytoplasm was stained, MMP-9 expression was considered positive. Regarding the critical value of positive expression, three studies included staining scores based on the intensity and percentage of stained cells, while other studies only used percentages.

\section{Study Quality}

We evaluated the quality of the studies based on a hypothetical ELCWP scale. The detailed scores are shown in Table 2. The overall average score of the study was $80.8 \%$. In all studies, the average score for research methods and analysis of resultswas 8.2 points, which was the same asthe score for design (8.2 
points) and higher than that forgenerality (7.7 points). The average total scores for invasion, relapse, and functional status were $78.5 \%, 83.3 \%$, and $83.3 \%$, respectively. According to a t-test, the difference between infiltration and recurrence was not statistically significant, indicating that the study baseline characteristics were homogeneous.

\section{Main Outcomes Of The Meta-analysis}

We classified pituitary adenomas as invasive and non-invasive. Twenty-two articles (Table 1) provided invasive status information, among which 514 patients with invasive pituitary adenomas had high MMP9 expression. As shown in Fig. 2, a fixed-effects model showed a significant correlation between MMP-9 expression and invasiveness $(n=22, O R=4.75,95 \% C l=3.53-6.39 ; p=0.000)$, while MMP-9 expression was also related to functional status $(n=3, O R=2.07,95 \% \mathrm{Cl}=1.03-4.16 ; p=0.041)$ through a fixed effect model. The correlations between MMP-9 and age and gender could not be determined due to insufficient sample size. The expression of MMP-9 in postoperative pituitary adenoma tissues has been suggested to allow prediction of the occurrence of invasive adenoma, and high expression of MMP-9 can be used as a clinical diagnosis marker.

In addition, three articles reported the value of MMP-9 overexpression with regard to determining the prognosis. After the meta-analysis, the expression of MMP-9 was significantly correlated with tumour recurrence $(n=3, O R=4.38,95 \% \mathrm{Cl}=1.76-10.94 ; p=0.002)$, (Fig. 3), suggesting that MMP-9 can effectively predict the tumour prognosis.

\section{Publication Bias And Sensitivity Analysis}

In this meta-analysis, according to the Begg's and Egger's test, 22 studies on invasion exhibited significant publication bias $(p=0.159,0.009)$, while MMP-9 and relapse or functional status studies did not show any $\operatorname{bias}(p=0.296,0.512 ; p=0.296,0.279)$. Similarly, the funnel plot revealed an asymmetric distribution of all intrusion studies (Fig. 4a), further demonstrating publication bias. In addition, a sensitivity analysis showed that no individual studies significantly affected the pooled ORs of MMP-9 and invasion status, suggesting that the results of this meta-analysis are robust. The detailed results are shown in Fig. 4b.

\section{Disussion}

Recent studies have shown that multiple biological and genetic variations of pituitary cancer cells may be one of the causes of a poor prognosis in patients with pituitary tumours ${ }^{[25]}$. Among them, MMP-9 is the most common factor due to direct invasion of surrounding tissues and blood vessels. Numerous studies have shown that MMP-9 plays an important role in tumour invasion and recurrence. Due to the important role of MMP-9 in tumour biology, it has been recommended as a prognostic factor and therapeutic target 
in other tumours ${ }^{[26,27]}$. At present, it is unclear whether MMP-9 can be used as an indicator of pituitary adenoma invasion and recurrence.

In this study, we investigated the effect of MMP-9 expression on the invasiveness and recurrence of 23 pituitary adenomas using large sample data. Here, the quality assessment was performed using a hypothetical ELCWP scale, which showed significant differences across all studies. If significant heterogeneity was observed among the studies, a random effects model, which provides a more conservative standard error and a larger $\mathrm{Cl}$, was chosen to determine the pooled OR /HR estimate. Our analysis showed that MMP-9 expression was significantly correlated with invasiveness $(n=22, O R=4.75$, $95 \% \mathrm{Cl}=3.53-6.39 ; \mathrm{p}=0.000)$ in 1153 patients. Using a fixed effect model, MMP-9 expression was also related to functional status $(n=3, O R=2.07,95 \% \mathrm{Cl}=1.03-4.16 ; p=0.041)$, but because of the small sample size, correlations between MMP-9 and age or gender could not be observed. In addition, the expression of MMP-9 was significantly related to tumour recurrence $(n=3, O R=4.38,95 \% \mathrm{Cl}=1.76-$ 10.94; $p=0.002$ ), (Fig. 3), suggesting that MMP-9 can effectively predict tumour recurrence. Clinical evidence is needed to provide effective treatment strategies to improve patient health. Our results confirm that MMP-9 is effective for predicting invasive pituitary adenoma in clinical practice.

Next, we used the classical Q test to assess heterogeneity. According to the criteria, when $p<0.10$ and/or $\mathrm{I}^{2}>50 \%$, heterogeneity was considered statistically significant. In this meta-analysis, no significant heterogeneitywas found among the studies of invasion, relapse, and functional status. Therefore, a fixed effects model was recommended in the analysis. However, several limitations of this study should be considered. First, the expression of MMP-9 in the Chinese population, which was examined most studies, was determined by IHC analysis. In general, IHC strongly relies on methodological factors such as primary and secondary antibody titres. However, it was very difficult to perform subgroup analyses based on different types of antibodies to investigate other possible biases. In addition, substantial differences in the cut-off values were observed among the studies. To date, no related studies have explored this hypothetical criterion for positive expression, which may be a potential source of bias. Unfortunately, most studies did not provide complete results and data, although this may not affect the bias.

In addition, publication bias is an important issue in systematic reviews, and it may cause bias. Most studies tend to report positive results, while studies with negative results are often rejected. In this study, both the Egger's and Begg's p-value tests and funnel plots implied publication bias. However, it should be noted that the languages of published articles included in this meta-analysis were limited to English and Chinese, which may cause publication bias regarding studies published in other languages that met our inclusion criteria.

\section{Conclusion}

In summary, MMP-9 expression is indeed related to tumour invasion and is an important factor in the recurrence of pituitary adenoma in patients. Therefore, the detection of MMP-9 in the pathological 
analysis will provide new ideas for accurately predicting the prognosis of patients, and it is also of great significance for selecting appropriate treatment methods.

\section{Methods}

\section{Search Strategy}

Literature searches were performed using the PubMed, Embase, Ovid, CNKI, and Wanfang databases through July 2019. No source or language restrictions were applied. The search terms included the following: "Matrix metalloproteinase 9"or "MMP9", "Pituitary adenoma", "Pituitary tumor", "Expression", "Invasive". All citations of the retrieved articles were scanned to identify other reports that may be available.

\section{Study Selection}

Two reviewers independently selected eligible studies. Any disagreements between the two reviewers were resolved through discussions with a third reviewer. The inclusion criteria were as follows: (1) pituitary adenomas confirmed by the pathology department; (2) the main research results focused on invasion, recurrence and hormone secretion; (3) immunohistochemical (IHC) identification of MMP-9 expression; (4) the hazard ratio (HR) and 95\% confidence interval (Cl)forMMP-9 expression and the biological behaviour of the tumour could be obtained directly from the article or calculated according to the graphs and tables given in the article; (5) for duplicate articles, only the most complete and/or recently published articles were used in the study.

\section{Data Extraction}

The following data were independently collected by two reviewers using specifically designed tables: first author name, year of publication, country, histology, research method, invasion, relapse, number of patients, mean age, cut-off value of stained cells, and positive rate. Any disagreements between the two reviewers were resolved by a third reviewer.

\section{Quality Assessment}

We evaluated the methodological quality of the included studies by reading and scoring each publication according to the Biological Prognostic Factor Quality Scale established by the European Lung Cancer Working Group (ELCWP) ${ }^{[28]}$. The scale assesses scientific design, laboratory methods, generality, and analysis of results. Each category can reach a maximum of 10 points, with a possible maximum value of 40 points. The two researchers compared their calculated scores and, if necessary, reached a consensus score for each category during a meeting. The final score represents the percentage of the maximum 
achievable points, ranging from 0 to $100 \%$. Therefore, higher values represent better methodological quality.

\section{Data Synthesis And Analysis}

The overall survival outcome data were synthesized using the prolonged HR as the operating index, Some included studies directly provided the HR and $95 \% \mathrm{Cl}$. For studies that did not directly provide the exact $\mathrm{HR}$ and $95 \% \mathrm{Cl}$, data were extracted from the original documentation, text or/and graphics, andSPSS13.0 software, Engauge Digitizer version 4.1 and the methods introduced by Tierney et al[29] and Parmar et al. ${ }^{[30]}$ were used to calculate these values.

To assess heterogeneity among the studies, we used the sum of squares $Q$ test. If the heterogeneity was significant $(p<0.1)$, a random effects model was used. Otherwise, we used a fixed effects model. A funnel plot created using the Begg's and Egger's tests was used to study publication bias ${ }^{\text {[31] }}$.

A sensitivity analysis was performed to verify the stability of the pooled results. The effect of MMP-9 on tumour invasion and recurrence was considered statistically significant if the corresponding $95 \% \mathrm{Cl}$ for the HR and/or OR did not overlap. The $p$ values were two-sided, and a value of $p<0.05$ was statistically significant. Statistical calculations were performed using Stata version 11.0 and RevMan version 5.0.

\section{Declarations}

\section{Ethics approval and consent to participate}

Not applicable

\section{Consent for publication}

Not applicable

\section{Availability of data and materials}

All data generated or analyzed during this study are included in this published article.

\section{Funding}

This study was supported by Beijing Science and Nature Fund(grant no.7172057). Scientific research and cultivation plan of Beijing Municipal Hospital(PX2018006)

\section{Competing Interests}

The authors declare that no competing interests exist.

\section{Authors' contributions}


Jun Kang designed and searched the articles. Bowen Sun and Congxin Dai analyzed and interpreted the data. Bowen Sun, Xiaohai Liu and Renzhi Wang were major contributors in writing the manuscript. The final version of the manuscript has been read and approved by all authors, and each author believes that the manuscript represents honest work.

\section{ACKNOWLEDGMENTS}

We greatly thank other members in our lab for their valuable suggestions and writing.

\section{References}

[1] Asa S L, Ezzat S. The pathogenesis of pituitary tumours[J]. Nat Rev Cancer, 2002,2(11):836-849.

[2] Ezzat S, Asa S L. Mechanisms of disease: The pathogenesis of pituitary tumors[J]. Nat Clin Pract Endocrinol Metab, 2006,2(4):220-230.

[3] Colao A, Grasso L F, Pivonello R, et al. Therapy of aggressive pituitary tumors[J]. Expert Opin Pharmacother, 2011,12(10):1561-1570.

[4] Patil C G, Prevedello D M, Lad S P, et al. Late recurrences of Cushing's disease after initial successful transsphenoidal surgery[J]. J Clin Endocrinol Metab, 2008,93(2):358-362.

[5] Kontogeorgos G. Predictive markers of pituitary adenoma behavior[J]. Neuroendocrinology, 2006,83(3-4):179-188.

[6] Herman V, Fagin J, Gonsky R, et al. Clonal origin of pituitary adenomas[J]. J Clin Endocrinol Metab, 1990,71(6):1427-1433.

[7] Wang E L, Qian Z R, Rahman M M, et al. Increased expression of HMGA1 correlates with tumour invasiveness and proliferation in human pituitary adenomas[J]. Histopathology, 2010,56(4):501-509.

[8] Tan H, Ye K, Wang Z, et al. CD147 expression as a significant prognostic factor in differentiated thyroid carcinoma[J]. Transl Res, 2008,152(3):143-149.

[9] Di leva A, Rotondo F, Syro L V, et al. Aggressive pituitary adenomas-diagnosis and emerging treatments[J]. Nat Rev Endocrinol, 2014,10(7):423-435.

[10] Stetler-Stevenson W G. Type IV collagenases in tumor invasion and metastasis[J]. Cancer Metastasis Rev, 1990,9(4):289-303.

[11] Roy R, Yang J, Moses M A. Matrix metalloproteinases as novel biomarkers and potential therapeutic targets in human cancer[J]. J Clin Oncol, 2009,27(31):5287-5297.

[12] Choi J Y, Jang Y S, Min S Y, et al. Overexpression of MMP-9 and HIF-1alpha in Breast Cancer Cells under Hypoxic Conditions[J]. J Breast Cancer, 2011,14(2):88-95. 
[13] Aalinkeel R, Nair B B, Reynolds J L, et al. Overexpression of MMP-9 contributes to invasiveness of prostate cancer cell line LNCaP[J]. Immunol Invest, 2011,40(5):447-464.

[14] Kumar B, Koul S, Petersen J, et al. p38 mitogen-activated protein kinase-driven MAPKAPK2 regulates invasion of bladder cancer by modulation of MMP-2 and MMP-9 activity[J]. Cancer Res, 2010,70(2):832841.

[15] Choe G, Park J K, Jouben-Steele L, et al. Active matrix metalloproteinase 9 expression is associated with primary glioblastoma subtype[J]. Clin Cancer Res, 2002,8(9):2894-2901.

[16] Wang $\mathrm{H}$, Wang $\mathrm{H}$, Jiao B. Expression of osteopontin, matrix metalloproteinase-9 in pituitary adenomas and the relationship between them in invasiveness[J]. Journal of Modern Oncology, 2013,21(05).

[17] Qiu L, He D, Fan X, et al. The expression of interleukin (IL)-17 and IL-17 receptor and MMP-9 in human pituitary adenomas[J]. Pituitary, 2011,14(3):266-275.

[18] Turner H E, Nagy Z, Esiri M M, et al. Role of matrix metalloproteinase 9 in pituitary tumor behavior[J]. J Clin Endocrinol Metab, 2000,85(8):2931-2935.

[19] Knappe U J, Hagel C, Lisboa B W, et al. Expression of serine proteases and metalloproteinases in human pituitary adenomas and anterior pituitary lobe tissue[J]. Acta Neuropathol, 2003,106(5):471-478.

[20] HE Dong-sheng C M W H, YAN Chao Z H H Y. Role of Matrix Metalloproteinases-9, 2 and Their Inhibitor-TIMP-1, 2 in Invasive Pituitary Adenomas Biological Behavior[J]. CHINESE JOURNAL OF CANCER, 2002,21(10):1124-1128.

[21] Xiang-dong L I, He-ping Z, Guo-zhen H, et al. Expressions of Ki-67 and MMP-9 and their relationship with the invasiveness in pituitary adenomas[J]. CHINESE JOURNAL OF NEUROSURGERY, 2008,24(10):762-764.

[22] Zhang H W R. Pituitary ACTH adenoma by adjusting factors associated with postoperative recurrence analysis - clinical review and immunohistochemical study[J]. Peking union medical college xue bao, 2009.

[23] Guangyu ZHANG, Baohua JIAO, Chaohui LIANG, \. The study on the association of MMP-9ロAng-1 and Ang-2 with tumor invasive behavior in human pituitary daenomas.[J]. CHINESE JOURNAL OF NERVOUS AND MENTAL DISEASES, 2011,37(6):333-336.

[24] Yubin LI. The Expression of Matrix metalloproteinase-2 $\varangle 9$ and their tissue inhibitor-1 $\varangle 2$ in human pituitary adenomas[J]. Zheng Zhou University, 2002.

[25] Heaney A. Management of aggressive pituitary adenomas and pituitary carcinomas[J]. J Neurooncol, 2014,117(3):459-468. 
[26] Jiang W G, Sanders A J, Katoh M, et al. Tissue invasion and metastasis: Molecular, biological and clinical perspectives[J]. Semin Cancer Biol, 2015,35 Suppl:S244-S275.

[27] Sato A, Nagase H, Obinata D, et al. Inhibition of MMP-9 using a pyrrole-imidazole polyamide reduces cell invasion in renal cell carcinoma[J]. Int J Oncol, 2013,43(5):1441-1446.

[28] Steels E, Paesmans M, Berghmans T, et al. Role of p53 as a prognostic factor for survival in lung cancer: a systematic review of the literature with a meta-analysis[J]. Eur Respir J, 2001,18(4):705-719.

[29] Tierney J F, Stewart L A, Ghersi D, et al. Practical methods for incorporating summary time-to-event data into meta-analysis[J]. Trials, 2007,8:16.

[30] Parmar M K, Torri V, Stewart L. Extracting summary statistics to perform meta-analyses of the published literature for survival endpoints[J]. Stat Med, 1998,17(24):2815-2834.

[31] Sterne J A, Egger M. Funnel plots for detecting bias in meta-analysis: guidelines on choice of axis[J]. J Clin Epidemiol, 2001,54(10):1046-1055.

[32] Gultekin G D, Cabuk B, Vural C, et al. Matrix metalloproteinase-9 and tissue inhibitor of matrix metalloproteinase-2: Prognostic biological markers in invasive prolactinomas[J]. J Clin Neurosci, 2015,22(8):1282-1287.

[33] LC G, YS Z, GZ H, et al. The clinical pathologic research of the pathological morphologic characteristics of invasive pituitary adenomas and its expression of Ki-67 and MMP-9[J]. Chin J Neurosurg, 2013,29(05).

[34] WANG Hui W H Z P. Expressions of osteopontin, matrix metalloproteinase-9 and microvessel density in pituitary adenomas and relationship

between them and pituitary adenomas invasiveness[J]. Chin J Clin Neurosurg, 2012,17(11):671-673.

[35] Dong Xiaohui W C. The expression and correlation study of COX-2,MMP-9 in invasive pituitary adenomas[J]. Chinese Journal of Clinical Rational Drug Use, 2011,5(9B):1-3.

[36] Cong-hui L I, Jian-ya Y E, Huan-li M, et al. Expressions of MMP-9, VEGF and p16 in human pituitary adenomas and their relationship with invasiveness of pituitary adenomas[J]. CHINESE JOURNAL OF CLINICAL NEUROSURGERY, 2010,15(7):409-412.

[37] Chao C. FAK and matrix metalloproteinases 9 expressed in pituitary adenomas[J]. NanChang University medical college, 2010.

[38] Guo-dong Q X Q Y. Expressions of CDI47هMMP-9 and VEGF in pituitary adenomas and relationship with biological behavior[J]. JOURNAL OF SHANDONG UNIVERSITY(HEALTH SCIENCES), 2008,46(3):288295. 
[39] Jiuzhou L I, Gang H, Luping Z, et al. The study on expression matrix metalloproteinase-9, HPA and CD34 in invasive pituitary adenomas[J]. CHINESE JOURNAL OF NERVOUS AND MENTAL DISEASES, 2007,33(7):404-407.

[40] Zhang Kun. The study of the Corrlation between invasiveness and expression of MMP.94VEGF and P16 in human pituitary adenomas[J]. Heibei Mdical University Xue Bao, 2007.

[41] Jian-nong Z, Jian W, Yun-sheng L, et al. Relationship between the expressions of PTTG, bFGF and MMP-9 and the invasiveness of pituitary adenomas[J]. CHINESE JOURNAL OF NEUROMEDICINE, 2007,6(3):282-285.

[42] Li Aijun W D W Y. Expressions of Pituitary Tumor Transforming Gene and Matrix Metalloproteinases in Pituitary Adenomas and Their Meanings[J]. CHINESE JOURNAL OF CLINICAL NEUROSURGERY, 2007,12(7):418-421.

[43] Jing-Lei W, Ting L. The Expression of MMP-2 and MMP-9 and Their Inhibiter TIMP-1 in the Invasiveness of Human Pituitary Adenoma[J]. JOURNAL OF CLINICAL EMERGENCY CALL, 2005,6(4):5-7.

[44] Liu W, Zhang X, YOSHIHITO. The biology behavior of matrix metalloproteinase-2 and 9 expression in invasive cavernous pituitary adenomas[J]. CHINESE JOURNAL OF NEUROSURGICAL DISEASE RESEARCH, 2004,3(1):47-51.

[45] Wang Zhen L C S H. Correlation of expression of MMP-9 and TIMP-1 with biological behaviors in pituitary adenomas[J]. CHINESE JOURNAL OF CLINICAL AND EXPERIMENTAL PATHOLOGY, 2004,20(2):200-202.

[46] Zhang Jianmin N H P S. Expression of matrix metalloproteinase in invasive pituitary adenoma and its relationship with microvessel density[J]. JOURNAL OF PRACTICAL ONCOLOGY, 2003,18(3):212-215.

\section{Tables}

Due to technical limitations, Tables 1-2 are provided in the Supplementary Files section.

\section{Figures}




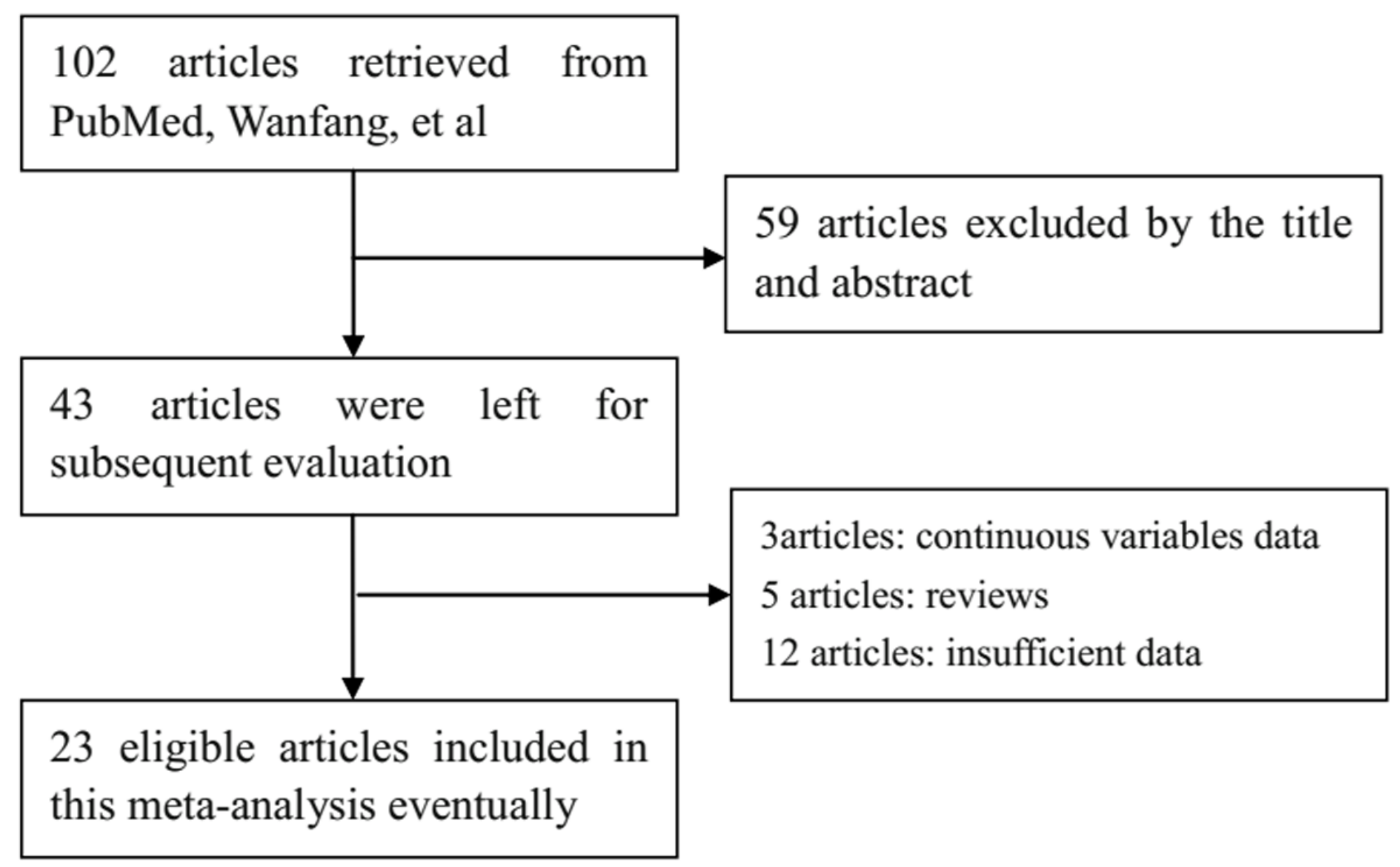

Figure 1

Literature search and selection of articles 


\begin{tabular}{|c|c|c|c|}
\hline \multicolumn{2}{|l|}{$\begin{array}{l}\text { Study } \\
\text { ID }\end{array}$} & \multirow{2}{*}{$\begin{array}{l}\text { OR }(95 \% \mathrm{Cl}) \\
8.50(2.04,35.46)\end{array}$} & \multirow{2}{*}{$\begin{array}{l}\% \\
\text { Weigh } \\
3.38\end{array}$} \\
\hline Qu Xin 2008 & 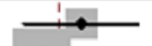 & & \\
\hline LIXD 2008 & & $3.09(125,7.65)$ & 12.62 \\
\hline Wu JL 2005 & & $8.57(0.83,89.04)$ & 1.32 \\
\hline liu WP 2004 & & $6.16(0.33,114.73)$ & 1.45 \\
\hline LiJZ 2007 & & $8.40(2.09,33.80)$ & 3.58 \\
\hline Zhang K 2007 & & $10.40(2.66,40.74)$ & 2.94 \\
\hline LiCH 2010 & & $17.21(0.90,328.82)$ & 0.82 \\
\hline Wang H 2012 & & $10.50(1.89,58.36)$ & 1.88 \\
\hline Zhao JN 2007 & & $21.00(2.87,153.75)$ & 0.94 \\
\hline Li AJ 2007 & & $6.58(0.71,61.08)$ & 1.79 \\
\hline Wang Z 2004 & & $7.33(0.81,66.63)$ & 1.80 \\
\hline Dong XH 2012 & & $6.20(1.87,20.59)$ & 5.14 \\
\hline LiYB 2002 & & $4.17(1.19,14.54)$ & 5.53 \\
\hline Zhang GY 2011 & & $4.50(0.50,40.65)$ & 2.35 \\
\hline He DS 2002 & & $0.76(0.03,16.86)$ & 2.33 \\
\hline Cheng C 2010 & & $5.44(1.58,18.71)$ & 5.07 \\
\hline Zhang JM 2003 & & $7.50(128,44.08)$ & 2.28 \\
\hline Turner 2000 & & $70.00(3.72,1317.87)$ & 0.25 \\
\hline Qiu LB 2011 & & $9.25(2.39,35.81)$ & 3.76 \\
\hline Ulrich J 2003 & & $0.86(0.34,2.15)$ & 23.05 \\
\hline Guo LC 2013 & & $2.82(1.14,6.98)$ & 13.22 \\
\hline Güliz 2015 & $\rightarrow$ & $6.00(1.70,21.21)$ & 4.53 \\
\hline Overall $(\mathrm{I}$-squared $=26.0 \%, \mathrm{p}=0.130$ ) & $\diamond$ & $4.75(3.53,6.39)$ & 100.00 \\
\hline $\begin{array}{c}1 \\
.00076\end{array}$ & & & \\
\hline
\end{tabular}

\section{a}

Study $\%$

ID $\quad$ OR $(95 \% \mathrm{CI}) \quad$ Weight

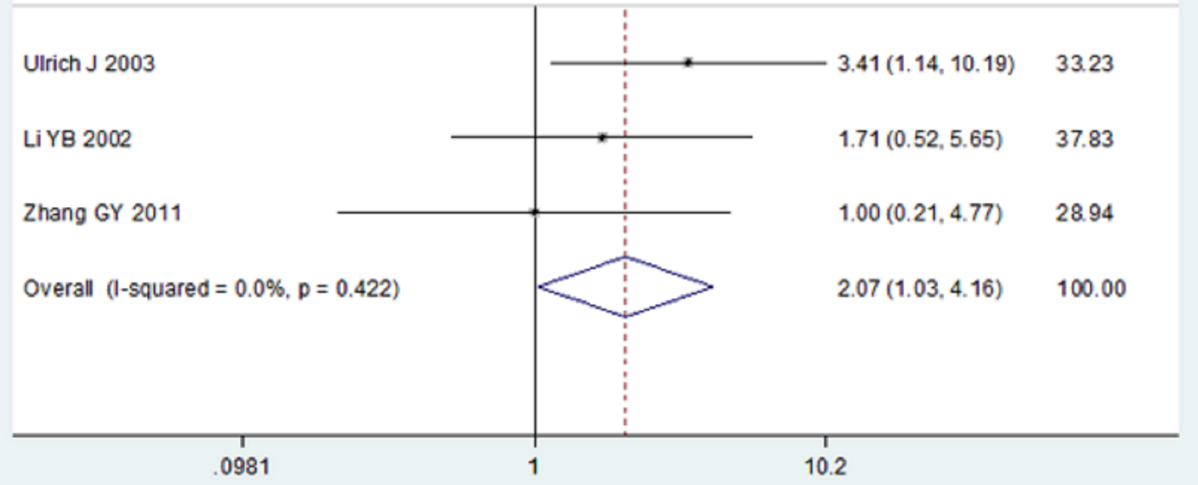

b

\section{Figure 2}

The individual and pooled ORs with 95\% ClsforMMP-9 andinvasiveness and functional status. A fixed effects model revealed an associationbetween MMP-9 and invasiveness $(n=22, O R=4.75,95 \% \mathrm{Cl}=3.53$ 6.39; $p=0.000)$, as well as between MMP-9 and functional status $(n=3, O R=2.07,95 \% \mathrm{Cl}=1.03-4.16$; $p=0.041)$ 
ID

OR $(95 \% \mathrm{Cl})$

Weight

LIXD 2008

Zhang H 2009

Turner 2000

Overall (I-squared $=0.0 \%, p=0.500)$

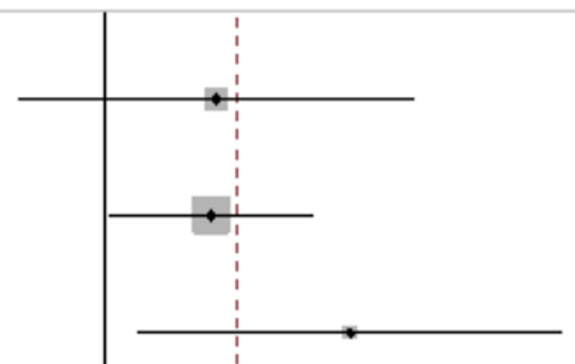

$3.48(0.38,31.63)$

22.72

$3.30(1.06,10.28) \quad 68.64$

$15.40(1.47,160.97) \quad 8.64$

$4.38(1.76,10.94) \quad 100.00$

\section{Figure 3}

The individual and pooled ORs with $95 \% \mathrm{Cl}$ for MMP-9 and recurrence. A fixed effects model revealed an associationbetween MMP-9 and recurrence $(n=3, O R=4.38,95 \% \mathrm{Cl}=1.76-10.94 ; \mathrm{p}=0.002)$
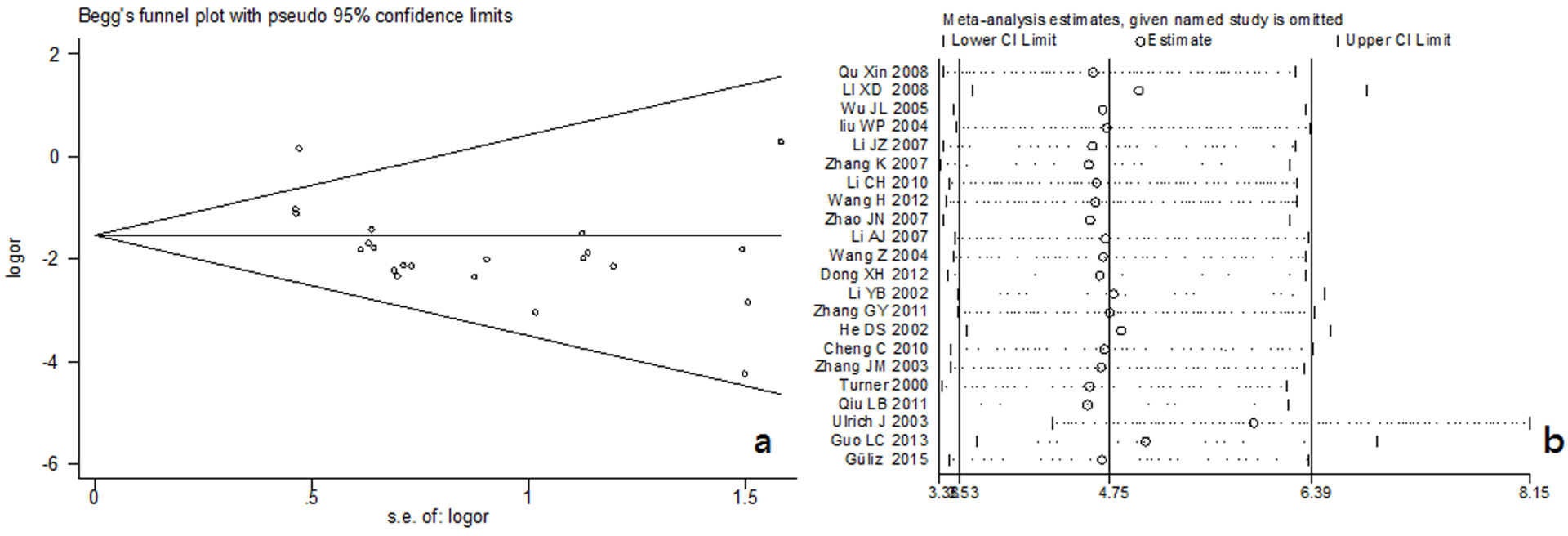

Figure 4 
Funnel plot and sensitivity analysis. A funnel plot was designed tovisualize potential publication bias. The shape of the funnel plotfor all studiesrevealed obvious evidence of asymmetry, suggestingthe existence of publication bias. A sensitivity analysis showed that all estimates (small cycle) were veryclose to the middle line.

\section{Supplementary Files}

This is a list of supplementary files associated with this preprint. Click to download.

- TABLE2.pdf

- TABLE1.pdf

- PRISMA2009checklist128.doc 